\title{
Essais
}

Revue interdisciplinaire d'Humanités

3 | 2013

Narration et lien social

\section{Pedro Alberto García Bilbao, Migraciones Forzosas: análisis de un concepto}

\section{Aubin Gonzalez}

\section{(2) OpenEdition}

1 Journals

Édition électronique

URL : https://journals.openedition.org/essais/9574

DOI : $10.4000 /$ essais. 9574

ISSN : 2276-0970

Éditeur

École doctorale Montaigne Humanités

\section{Édition imprimée}

Date de publication : 15 mai 2013

Pagination : 218-221

ISBN : 978-2-9544269-0-7

ISSN : 2417-4211

\section{Référence électronique}

Aubin Gonzalez, "Pedro Alberto García Bilbao, Migraciones Forzosas: análisis de un concepto », Essais [En ligne], 3 | 2013, mis en ligne le 01 septembre 2021, consulté le 08 septembre 2021. URL : http:// journals.openedition.org/essais/9574; DOI : https://doi.org/10.4000/essais.9574 
Pedro Alberto García Bilbao, Migraciones Forzosas: análisis de un concepto, Silente Academica, Madrid, 2011, 94 p.

Cet ouvrage du sociologue Pedro Alberto Garcia Bilbao propose d'analyser le concept de " migration forcées " depuis la perspective globale des sciences sociales. L'objectif est clair : il s'agit d'identifier d'emblée ce dont on parle lorsqu'on se consacre à l'histoire et aux théories migratoires. En effet, les études migratoires constituent un champ de recherche avec une singularité qui repose sur la transdisciplinarité au sein même des sciences sociales. Cependant, cette dimension transdisciplinaire soulève de sérieuses questions quant aux usages du concept de migrations et aux catégories qu'il met en jeu. Outre le fait d'entendre la "migration " comme un processus de mobilité qui, de façon analogue, présente un caractère exclusif et inclusif, l'analyse du concept met en relief une tension entre deux objets : les individus et la dimension collective d'un phénomène migratoire.

Ceci, car la migration traduit généralement un phénomène de déplacement de populations en masse, recoupant ainsi la définition de ce que l'on appelle la « déportation » ou encore l'« exil ». Cette manière d'entendre le concept suppose part ailleurs d'ôter toute volonté aux sujets dans le processus de mobilité, alors même que " émigration " et " immigration " tendent à démontrer le contraire dans leurs acceptions. Loin de restreindre cette mobilité à un cadre géographique, c'est en rappelant les apports de la sociologie, de la démographie ou de la géographie humaine que le "déplacement d'un groupe ou d'un individus " s'explique aussi en terme de mobilité sociale, internationale, intrarégionale, etc. Dès lors, qui s'intéresse aux théories migratoires se trouve confronté à un objet hybride et surtout difficile à cerner ${ }^{2}$. Pour Garcia Bilbao, identifier ce dont nous parlons signifie donc gagner d'avantage en précision topologique. La compréhension évidente des modalités de chaque mobilité permet en outre de rendre les catégories avec lesquelles on désigne les vagues migratoires plus ou moins opérantes suivant les cas de figure.

Ainsi, certains de ces cas, comme l'exil par exemple, présenterait un caractère obligatoire et involontaire à la différence d'autres cas (migrations du travail), où la motivation individuelle et/ou collective serait manifeste. Cette distinction, qui est d'ailleurs reprise par les institutions internationales, et les nuances qu'elle peut présenter correspondent aux catégories de vagues migratoires. Cependant, il semble que cette distinction catégorielle entre migrations

2 L'un des cas les plus significatif à nos yeux reste les problèmes posés par la vague de migrations intérieures dans l'Europe contemporaine. Citons à titre d'exemple les travaux de Maurizio Gribaudi, Itinéraires ouvriers, Espaces et Groupes Sociaux à Turin, au début du XXe siècle, EHESS, Paris, 1987 ; Marin Corbera Marti, Memories del Viatge (1940-1975), MHIC, Sant Ádria de Besós, 2009 ; Noiriel Gérard, Immigration, Antisémitisme et Racisme en France : discours publics, humiliations privés, XIX-XXe siècle, Hachette, Paris, 2007. 
volontaires et involontaires soit surtout opératoire depuis le strict point de vue des migrations entendues comme le déplacement massif d'individus ${ }^{3}$. De ce fait, on tend logiquement à écarter de façon significative la place des sujets individuels dans ces vagues. Aussi convient-il d'emprunter un aspect significatif de l'historiographie espagnole pour illustrer notre propos.

Pendant longtemps en effet, certains auteurs, fleurons de l'histoire économique espagnole, ont justifié l'arrivée massive d'exilés ruraux dans les grandes villes du pays comme une conséquence du développement économique de l'Espagne $e^{4}$. Or, de récentes recherches viennent invalider cette hypothèse. Non seulement, l'arrivée massive d'individus dans les grandes villes et agglomérations du pays accentue la catastrophe économique des années d'après guerre civile, mais pire encore : elle montre à quel point la vague de migrations intérieure des années cinquante présente un caractère inévitable !' Inévitable pour qui veut échapper au régime franquiste sous lequel la répression est une véritable institution. Inévitable parce que la survie du groupe ou de l'individu en dépend. N'est-ce pas là une contrainte où la motivation n'est pas nécessairement exogène?

En outre, dans quelle catégorie classer ce cas de figure ? C'est une question d'autant plus sérieuse quand on sait que le franquisme n'a cessé de vouloir contrôler ces afflux de personnes. Les migrations intérieures ne présentent pas un caractère " obligatoire " mais sont bel et bien "inévitables " par ce qu'elles impliquent. C'est pourquoi le concept de migrations " contraintes » obéit, selon l'auteur de l'ouvrage qui nous occupe, à une autre catégorie. Une catégorie qui, à la différence des autres que l'on a mentionnées ici, possède une " capacité d'adaptation " à l'échelle d'un groupe ou d'un individu. Fidèle à l'esprit de synthèse qui caractérise cet ouvrage, un certain parti pris émerge lorsque Garcia Bilbao reprend à son compte la définition selon laquelle :

"La migration contrainte est un mode de réponse face à un stimulus externe capable d'annuler la volonté de demeurer dans son propre milieu, faisant de cette dernière un élément dérisoire face à la nécessité de survivre ". (Garcia Bilbao, p. 65)

Ceci étant, l'intérêt pour les modalités migratoires s'avère être également un excellent moyen d'appréhender le concept autrement que par la question de l'usage des catégories. En effet, cela constitue un autre élément du travail

3 Zelinsky Wilbur, "The hypotesis of the mobility transition", in Geographical Review, vol. 61, $\mathrm{n}^{\circ} 2,1971$, p. 219-249.

4 Garcia Barbancho, Alfonso, Las Migraciones Interiores españolas: un estudio cuantitativo desde 1900, Publicaciones del Instituto del Desarrollo Económico, Madrid, 1967.

5 Marin Corbera, Marti, Memories del viatge, op. cit.

6 L'auteur reprend ici la thèse de Irantzu Mendia proposée dans l'article " Migraciones Forzosas ", in Peréz de Arminio, Karlos, Diccionario de Acción Humanitaria y Cooperacion al Desarrollo, Hegoa, Bilbao, 2000, p. 364-366. 
de synthèse que nous propose Garcia Bilbao mais qui requiert de considérer avec un œil neuf les dynamiques migratoires. C'est là ce qu'a proposé le Haut Commissariat aux Réfugiés des Nations Unies en mobilisant le lexique du travail humanitaire. Ceci étant, c'est encore oublier une catégorie, même s'il prétend suivre l'évolution des groupes ou individus dont il assure la protection au-delà du simple statut de réfugié. La proposition du HCR nous ramène donc au constat de départ : celui d'une migration toujours vue comme un déplacement massive et involontaire qui ne prend pas en compte la mobilité sociale. (Garcia Bilbao, ch. 8)

Le dernier chapitre de cette synthèse a par conséquent l'objectif de présenter une nouvelle définition du concept de migration "contrainte ", dans le tournant de l'approche des sciences sociales autour de ce terme. Avec la définition proposée, les " migrations contraintes " deviennent l'objet d'une nouvelle catégorie propre à l'étude des pratiques et théories migratoires. Cet objet nécessite une attention particulière dans son approche parce que la dynamique migratoire dépasse de fait la mobilité dans son ensemble. Dans leurs dynamiques, les migrations contraintes sont aussi des moteurs de transformation des cadres sociaux à l'intérieur desquels les groupes ou individus évoluent. Ces cadres sociaux pouvant être régionaux, nationaux et internationaux, ils peuvent être par ailleurs soumis à différents moyens de coercition qui laissent plus ou moins de place à une réponse du ou des groupes sociaux. Ainsi, la définition énoncée en fin d'ouvrage contribue à réactualiser le concept de migration et à réactualiser dans le même temps la démarche des sciences sociales dans son intérêt pour les études migratoires.

Une réactualisation, certes, mais à nuancer tout de même. Bien qu'elle soit précédée d'une excellente synthèse des différentes approches que permettent les sciences sociales, la perspective historique semble être mise de côté ou partiellement affirmée. En effet, la révision constante du concept de migration depuis la fin du XIX siècle et durant tout le $\mathrm{XX}^{\mathrm{e}}$ siècle contribue à mesurer la portée de phénomènes migratoires méconnus jusqu'alors. Aussi pouvons nous penser que les migrations est un objet d'histoire récent et, qui plus est encore en construction. À la lumière des différents travaux réalisés sur le sujet, reconnaissons que l'appropriation du concept de migration par la discipline historique est un fait propre de l'histoire contemporaine. En outre, des historiens comme Noiriel ou Bade ont porté un intérêt aux migrations, en tant qu'elles permettent de penser la construction de l'identité d'un groupe ${ }^{7}$. Il a donc fallu des travaux comme ceux proposés par Nancy Green pour repenser les migrations, comme un objet d'histoire à part entière et plus seulement en

7 Jurgen Bade, Klaus, L'Europe en Mouvement : de la fin du XVIII' siècle à nos jours, Éd. du Seuil, Paris, 2002 ; Noiriel, Gérard, op. cit. 
tant que stratégie intellectuelle ${ }^{8}$. Est-il également besoin de souligner l'apport de l'histoire transnationale dans ce processus d'appropriation car il y a des phénomènes historiques qui la notion même de frontière, qui apparait elle même comme un objet à déconstruire. On reconnaît nettement l'ambition affichée dans cet ouvrage qui est de faire l'histoire de ce concept. Cependant, si cela n'est pas explicitement formulé par l'auteur, force est de constater qu'il s'agit d'un aveu à demi mot. Sans doute cela s'explique t-il par une prudence qui voudrait que le sociologue n'empiète pas sur les plates bandes de l'historien. C'est pourtant Garcia Bilbao lui-même qui, dans ses pages rappelle, la nécessité d'approcher le concept de migration « contraintes » sous l'angle de la transdisciplinarité ! (Garcia Bilbao, ch. 6)

Aubin Gonzalez

EA $4574 \mathrm{SPH}$

Université Michel de Montaigne Bordeaux 3

aubin.gonzalez@etu.u-bordeaux3.fr

\begin{abstract}
François Trahais, Catalogue d'exposition réalisé par Susan Hapgood, Cornelia Lauf (éd.), In deep: certificates of authenticity in art, Roma publications, Amsterdam et SBKM / De Vleeshal, Middelburg, 2011. Langue : anglais, $102 \mathrm{p}$.
\end{abstract}

Pour la première fois dans l'histoire des catalogues d'exposition, un recensement exhaustif des certificats d'authenticité dans l'art du $\mathrm{XX}^{\mathrm{e}}$ siècle vient d'être édité. Témoignant d'une exposition ayant récemment circulé à travers l'Europe, les États-Unis et l'Asie, l'ouvrage se caractérise par la richesse de ses illustrations en couleur. De manière inédite, un corpus de cinquantedeux contrats de cession, protocoles de réalisation et de certificats d'authenticité y est présenté. Ces " énoncés d'art " tel que les nomment de manière générique Ghislain Mollet-Viéville et Jean-Baptiste Farkas', s’inscrivent dans l'histoire de la dématérialisation de l'œuvre. Avec l'invention du readymade par Marcel Duchamp au début du siècle dernier et le développement d'une certaine forme de conceptualisme, l'idée prime désormais sur l'objet dans l'histoire de la modernité. D’autant plus que les nouvelles techniques de reproductibilité de l'œuvre contribuent elles aussi à discuter les traditionnels critères d'authenticité. Les conditions de matérialité et d'originalité sont alors remises en question. Le statut de l'auteur quant à lui se repense en fonction de la participation et de

8 Selon le titre de l'ouvrage de Nancy Green, Repenser les Migrations, PUF, Paris, 2002.

9 G. Mollet-Viéville \& J.-B. Farkas, "À propos des "énoncés d'art" ", in Critique, Les Édition de Minuit, août-septembre 2010, p. 719-734. 